\title{
Is local hypoperfusion the reason for transient neurological deficits after STA-MCA bypass for moyamoya disease?
}

\author{
Nitin Mukerji, MSc, MD, FRCSEd, Douglas J. Cook, MD, FRCSC, and Gary K. Steinberg, MD, PhD \\ Department of Neurosurgery and Stanford Stroke Center, Stanford University School of Medicine, Stanford, California
}

\begin{abstract}
OBJECT Hyperperfusion is believed to be the cause of transient neurological events (TNEs) in patients with moyamoya disease (MMD) who have undergone an extracranial-to-intracranial (EC-IC) bypass between the superficial temporal artery (STA) and the middle cerebral artery (MCA). The objective of this study was to evaluate this possibility by analyzing cerebral blood flow (CBF) data obtained with thermal diffusion probes used at the authors' center.

METHODS The authors examined postoperative cerebral perfusion in 31 patients with MMD who underwent a direct EC-IC STA-MCA bypass. A Hemedex Q500 flow probe was placed in the frontal lobe adjacent to the bypass and connected to a Bowman cerebral perfusion monitor, and CBF data were statistically analyzed using JMP 8.0.2 software. Seven patients experienced a TNE after surgery in the left hemisphere (that is, after left-sided surgery), manifesting as dysphasia approximately 24 hours postoperatively and which had improved by 48 hours. No TNEs were observed after right-sided surgeries. Operative and postoperative CBFs in the left side with the TNE were compared with those in the left side with no TNE and on the right side.
\end{abstract}

RESULTS A detailed analysis of 64,980 minute-by-minute flow observations showed that the initial postbypass CBF was higher on the left side where the TNEs occurred. This CBF increase was followed by a widely fluctuating pattern and a statistically significant and sharp drop in perfusion ( $p<0.001$, mean difference of CBF between groups, paired t-test) associated with a TNE not observed in the other 2 groups.

CONCLUSIONS On the basis of the authors' initial observations, an early-onset altered pattern of CBF was identified. These findings suggest local hypoperfusion as the cause of the TNEs. This hypoperfusion may originate from competing blood flows resulting from impaired cerebral autoregulation and a fluctuating flow in cerebral microcirculation.

http://thejns.org/doi/abs/10.3171/2014.8.JNS132413

KEY WORDS moyamoya; EC-IC bypass; hyperperfusion; hypoperfusion; vascular disorders

A

$\mathrm{N}$ extracranial-to-intracranial (EC-IC) bypass increases the vascularity of the cerebral hemisphere in patients with moyamoya disease (MMD) or its variants $^{8,11}$ and is widely used as the treatment of choice for symptomatic MMD. . $^{3-5,11,14}$ Numerous studies have shown that EC-IC bypasses decrease the risk of future stroke and transient ischemic attacks compared with medical therapy alone., ${ }^{3,14}$ Transient neurological events (TNEs) may occur after an EC-IC bypass, and the incidence of such events varies among different reports. The TNEs are generally attributable to hyperperfusion after the bypass, ${ }^{1,2,7,9,10}$ supported by evidence from both immediate intraoperative flow and postoperative imaging data. These data indicate a markedly increased flow in the ischemic cerebral hemisphere. However, no specific re- ports of real-time, minute-by-minute flow patterns in the operated hemisphere have been reported in the literature. Here, we describe our observations in 7 patients, each of whom had a bilateral EC-IC bypass for MMD and experienced a TNE after a surgery in the left hemisphere (that is, after left-sided surgery). We hypothesized that the origin of the TNEs may be a combination of the impact of initial hyperperfusion and large fluctuations in perfusion, leading to local and transient hypoperfusion.

\section{Methods}

\section{Patient Selection and Intraoperative Data Collection}

In this study, we included 31 consecutive patients with MMD who underwent a direct superficial temporal artery

ABBREVIATIONS CBF = cerebral blood flow; EC-IC = extracranial-to-intracranial; $I Q R=$ interquartile range; $M A P=$ mean arterial pressure; $M C A=$ middle cerebral artery; MMD = moyamoya disease; $S T A=$ superficial temporal artery; TNE = transient neurological event.

SUBMITTED October 29, 2013. ACCEPTED August 29, 2014.

INCLUDE WHEN CITING Published online October 24, 2014; DOI: 10.3171/2014.8.JNS132413.

DISCLOSURE This study was supported in part by funding from Josef Huber Family Moyamoya Fund, Stanley and Alexis Shin, and the Reddy Lee Moyamoya Fund (to G.K.S.). Dr. Steinberg is a member of the Medtronic Neuroscience Strategic Advisory Board and a consultant for Medtronic. 
(STA) to middle cerebral artery (MCA) bypass and whose postoperative perfusion was monitored. To measure cerebral blood flow (CBF), a Q500 thermal diffusion probe (Hemedex) was placed in the frontal lobe adjacent to the bypass and connected to a Bowman perfusion monitor (Hemedex). The intraoperative CBF was measured using a Doppler flowmeter (Transonic). The CBF data were recorded on a minute-by-minute chart with no inbuilt smoothing. Some of the patients undergoing a left-sided surgery experienced a TNE. These TNEs manifested as an onset of dysphasia approximately 18-24 hours after the operation and lasted for up to 96 hours.

For detailed analysis of these events, we divided all 31 patients into 3 groups: left-sided surgery with TNE (Group 1), left-sided surgery with no TNE (Group 2), and right-sided surgery (Group 3). Intraoperative measurements of variables, including blood flow in the anastomosed arteries, were then compared among the 3 groups, with Groups 2 and 3 serving as controls. This accounted for the possibility that the right-sided circulation was intrinsically different from the left-sided one, suggested by our observation that during the course of this study no TNEs occurred after surgery in the right hemisphere. The data were examined for significant differences in $\mathrm{CBF}$ among the groups at various times in the postoperative period.

\section{Statistical Analysis}

Raw data from minute-by-minute recordings of CBF, along with patient demographics and intraoperative data of the blood flows, were exported as text and spreadsheets and analyzed using JMP 8.0.2 software (SAS Inc.). The patients were classified into 3 groups as described above, and the intraoperative parameters were statistically compared among the 3 groups using ANOVA. Eight-hour time stamps were imposed on the CBF observations with the first recorded observation being labeled as 1. Data from observations for each time stamp were pooled within the 3 groups, and the medians of CBF at each time stamp were used to account for skewed distributions caused by outliers. The patterns of changes in median CBFs over time were plotted for the 3 groups with smoothing by the moving-average method. The analysis was limited to the first 50 hours after the operation, and the observations were divided into five 8 -hour time periods: Period 1, $0-8$ hours; Period 2, 8-16 hours; Period 3, 16-24 hours; Period 4, 24-32 hours; and Period 5, $\geq 32$ hours.

The differences in the median blood flow among the various time periods and among the groups were statistically analyzed to determine the significance of these differences. Significant differences were correlated with clinical observations.

\section{Results}

The group of patients in our study comprised 24 women and 7 men with a median age of 32 years (interquartile range [IQR] 25-46 years). All patients showed symptoms of MMD, confirmed by angiography, with evidence of ongoing ischemia for which they underwent direct revascularization. In total, 51 operations were performed; 20 of these were bilateral and 11 unilateral. Of these, 25 were right sided with no TNEs, 19 were left sided with no TNEs, and 7 were left sided with a TNE.

Intraoperative details of $\mathrm{CBF}$, occlusion time, mean arterial pressure (MAP), and end-tidal $\mathrm{CO}_{2}$ are shown in Table 1. No significant differences in baseline flow patterns in the MCA were observed between the 2 hemispheres. On the side of the TNE, the postoperative MCA flow was higher both antegrade and retrograde in the distal and proximal MCAs, respectively, but this difference did not reach statistical significance. No statistically significant differences were observed, and the occlusion times and end-tidal $\mathrm{CO}_{2}$ readings were similar for both sides.

On average, 20 hours (IQR 10-32 hours) of postoperative $\mathrm{CBF}$ data were available for each patient. The maximum recording lasted 50 hours after the operation, and the analysis was therefore limited to this time period. Detailed results from the analysis of 64,980 minute-by-minute flow recordings from the 3 groups and the moving-average plots for the changes in pooled median perfusions over time are shown in Fig. 1. The flow pattern appeared as an oscillating and widely variable pattern; this was much more evident in the group with the TNEs (that is, Group 1). Group 1 started off with higher perfusions, but showed very significant decreases around the time of the TNEs (Fig. 1A).

The differences in the means of the pooled median perfusions among the 3 groups are shown in Table 2. It was evident that for each of the 8-hour time periods the CBFs in the patients of Group 1 were significantly different from the CBFs in the other 2 groups. When the median perfusions were analyzed within each group, they were significantly different at the different time periods, most evident in Group 1, implying a highly variable flow pattern in this group.

\section{Discussion}

Methods for the continuous measurement of $\mathrm{CBF}$ are now available. One such method is the use of a thermal perfusion probe such as the QFlow 500 probe used here, which is placed intracerebrally in the vascular area of interest. To display the CBF data, the probe is connected to a monitor such as the Bowman perfusion monitor (Model 500) used in this study. With this setup, clinicians can measure continuous real-time CBFs to identify cerebral edemas and evaluate how the blood flows respond to therapies. The setup also enables studying time courses of CBF patterns. Such time courses can be accurately determined with the continuous time-series data provided by this system.

The current literature on thermal perfusion probes has focused on the clinical feasibility and technical capabilities of these probes. Jaeger et al. ${ }^{6}$ demonstrated that the use of thermal perfusion probes is reliable and that its results correlate well with those of other methods for measuring blood flow. Vajkoczy et al. ${ }^{13}$ have also validated this approach and noted that the values recorded by the flow probe show a drift with time requiring a statistical correction to account for this drift. Rosenthal et al. ${ }^{12}$ used this probe in patients with traumatic brain injury as part of multimodality monitoring.

The reported use of thermal diffusion probes in the set- 
TABLE 1. Details of the intraoperative parameters for the 3 patient groups*

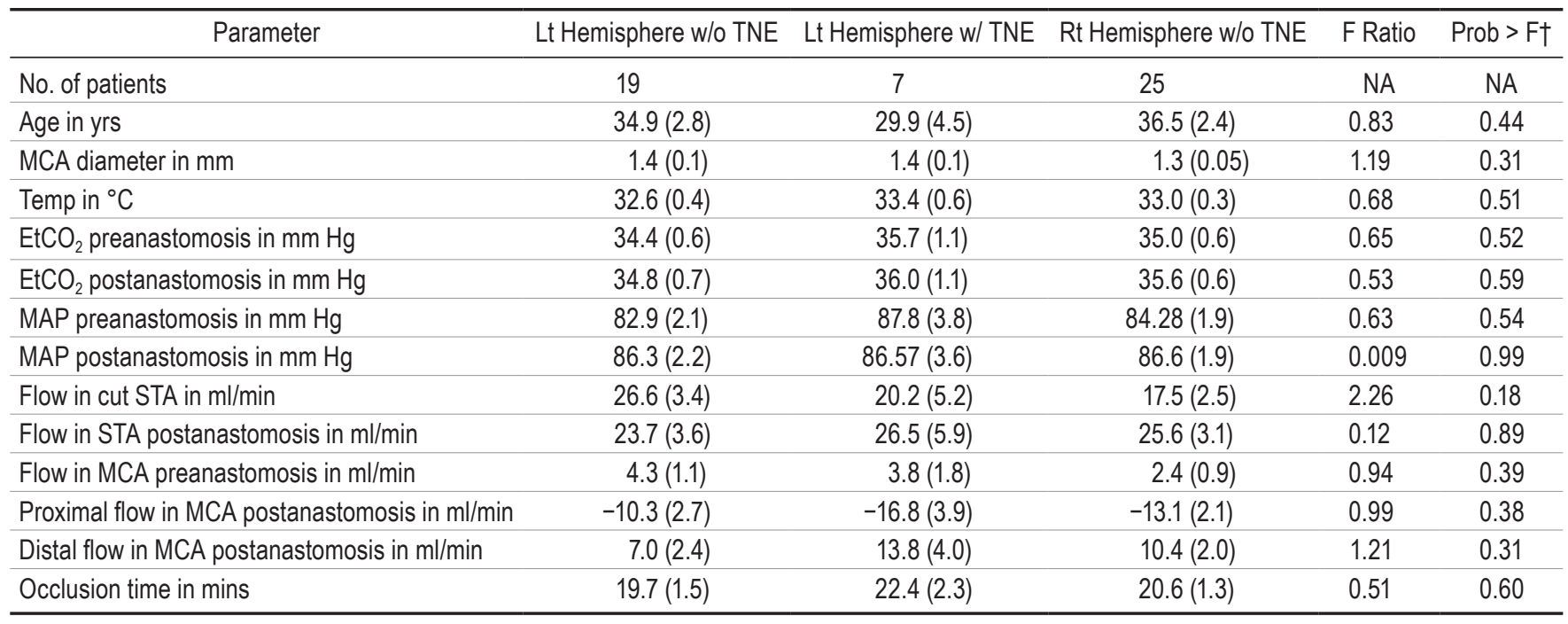

$\mathrm{EtCO}_{2}=$ end-tidal carbon dioxide concentration; NA = not applicable.

* Except for the first row and the last 2 columns, values are presented as the mean (SEM).

$\dagger$ Smaller values of the Prob $>F$ value indicate at least 1 significant effect in the statistical model.

A
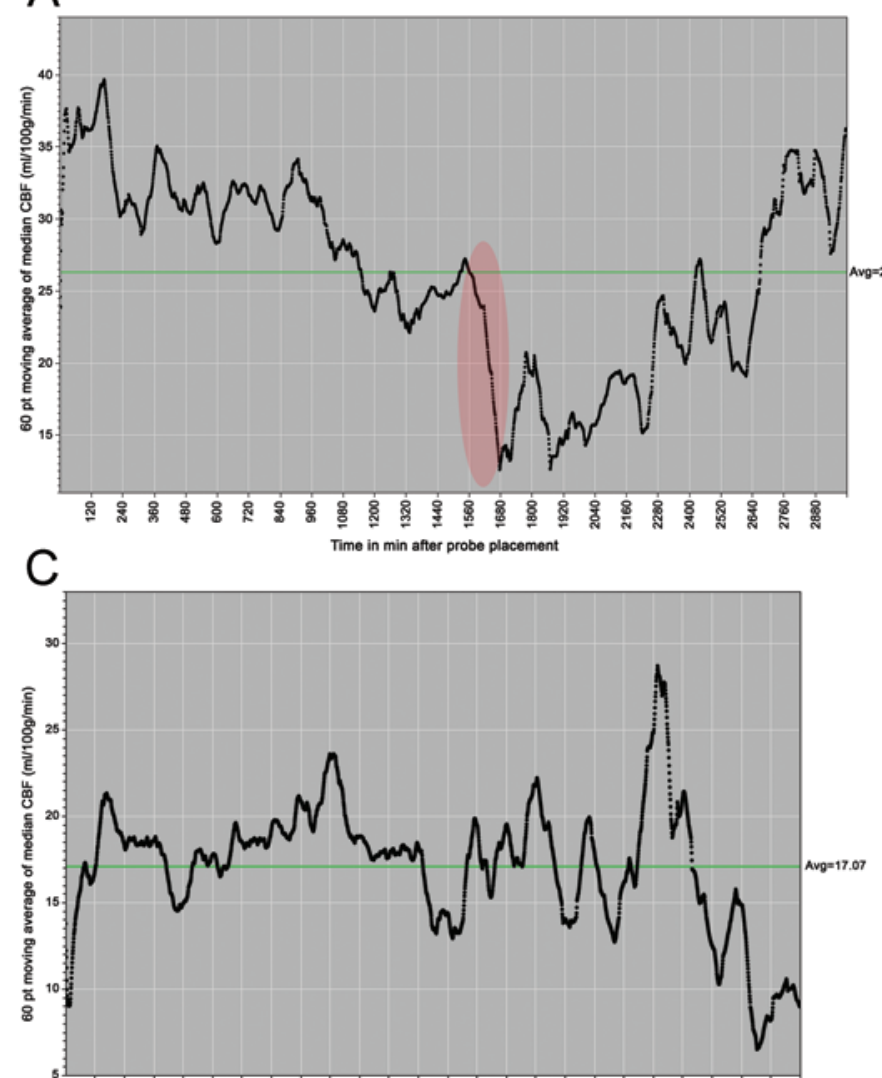

B

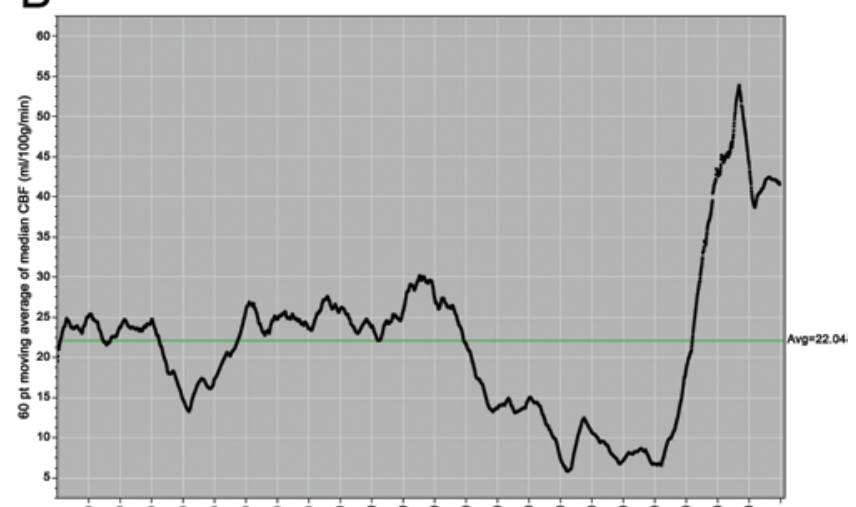

๕

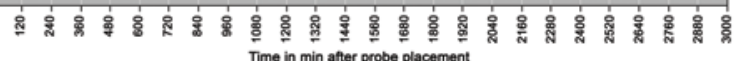

FIG. 1. Moving-average plots of pooled median perfusion over time in Group 1 (left-sided surgery with a TNE; the red-shaded oval marks the time period during which the TNE occurred) (A), in Group 2 (left-sided surgery with no TNE) (B), and in Group 3 (rightsided surgery with no TNE) (C). Figure is available in color online only. 
TABLE 2. Differences between Group 1 and the other 2 groups in median CBFs pooled in 8-hour time periods*

\begin{tabular}{ccc}
\hline & \multicolumn{2}{c}{ Mean Difference } \\
\cline { 2 - 3 } Time Period (hrs) & $\begin{array}{c}\text { Group 2 vs Group 1 } \\
\text { (ml/100 g/min) }\end{array}$ & $\begin{array}{c}\text { Group 3 vs Group 1 } \\
\text { (ml/100 g/min) }\end{array}$ \\
\hline $0-8$ & -11.41 & -15.91 \\
\hline $8-16$ & -9.34 & -12.84 \\
\hline $16-24$ & 0.23 & -6.42 \\
\hline $24-32$ & -3.39 & -2.37 \\
\hline$>32$ & -1.72 & -8.87 \\
\hline
\end{tabular}

* Group 1 had left-sided surgery with a TNE, Group 2 left-sided surgery with no TNE, and Group 3 had right-sided surgery with no TNE. All CBF differences shown were statistically significant ( $p<0.001$, paired t-tests).

ting of MMD and EC-IC bypasses has been very limited, despite the significant clinical potential of this approach. Prospective clinical studies are needed to determine the clinical value of thermal perfusion probes. This requires comparing the clinical outcomes of using these probes with the outcomes of other standard methods for identifying individuals at risk for secondary ischemic brain injury or reperfusion injury (for example, in traumatic brain injury, subarachnoid hemorrhage, or after EC-IC bypass) and for monitoring $\mathrm{CBF}$ responses to therapeutic interventions.

We have used thermal perfusion probes in patients with MMD who underwent an EC-IC bypass and report our experience here. Our data indicate that patients with TNEs (that is, Group 1) had a higher initial postbypass perfusion. Given that cerebrovascular reactivity is chronically impaired in these patients, such a large flow could result in hyperperfusion. A review of the trend of flow (Fig. 1A) confirmed that the cerebral autoregulation was indeed impaired in these patients. Especially in the patients of Group 1-that is, those who underwent surgery in the left hemisphere and experienced a TNE-the flow displayed a high intrinsic variability, indicating that the cerebral microcirculation in these patients failed to maintain a normal or steady flow.

The observed trends demonstrated that $\mathrm{CBF}$ after revascularization was not consistently high, but followed a variable pattern with significant drops in regional $\mathrm{CBF}$ between 12 and 24 hours postsurgery and again at 36 hours postsurgery. These drops also correlated temporally with the onset of TNEs (Fig. 1A). We therefore hypothesize that a combination of competing blood flows from the collateral circulation, new blood flow from the STA, and impaired cerebral autoregulation led to episodic focal decreases in regional CBF. This decrease in regional $\mathrm{CBF}$, in turn, caused the observed neurological deficits. Lee et al. ${ }^{8}$ have previously shown that following revascularization, the intraoperative postbypass $\mathrm{M}_{4}$ segment flow (combined proximal and distal to the anastomosis) can increase up to 5-fold, and that patients who develop a TNE have significantly higher postbypass $\mathrm{M}_{4}$ and STA flows than patients who do not develop TNEs.

We analyzed CBF data for a maximum of 50 hours postsurgery for the patients with TNEs, and it is difficult to extrapolate from these data the further development of the CBF in these patients. However, because these neurological deficits typically improve after $48-72$ hours, it is likely that the CBFs had normalized to a higher level by this time. We believe that postbypass hyperperfusion is a significant risk, but so is local hypoperfusion, and both can lead to TNEs.

Our data have important implications for patient management. Rather than arbitrarily maintaining the blood pressure between certain parameters as is normally recommended, it seems more reasonable to manage blood pressure by titrating it, using real-time data. Since our continuous CBF data suggest hypoperfusion at the onset of a TNE, to raise the blood pressure and increase perfusion, we treat these patients with midodrine, intravenous fluids, and sometimes fludrocortisone.

The small number of patients with TNEs limits our report; nonetheless, we were able to detect significant changes in local perfusion in these patients compared with those without a TNE. Some data were missing due to machine recalibrations, and these data were smoothed over by a standard moving-averages method. While we have retrospectively identified clear differences in $\mathrm{CBF}$ among the groups, it may be difficult to accurately model and predict which patients will develop TNEs. It was clear that the Group 1 patients started with higher flows, had greater fluctuations, and experienced a greater drop in flow when the TNE became clinically apparent.

Further studies and correlations of CBF data with those from MRI, the preoperative status of autoregulation, and postoperative MAPs would be helpful. Limitations in machine design and in the data obtained should be addressed, because frequent recalibration leads to missing data, and data variability increases with changes in brain temperature, especially in the immediate postoperative period when the brain temperature is raised from intraoperatively induced mild hypothermia. These missing CBF values weaken the value of the continuous timeseries data collected with the probe because converting continuous data to discrete values diminishes the power of the longitudinal analysis. An unsmoothed plot as read on the monitor can be misleading at times, and a trend of reduced CBF may be difficult to detect. However, we note that well-defined drops in flow are well displayed. The intrinsic variability of the data is demonstrated in Fig. 2, providing a raw snapshot from 1 patient with a TNE.

It is important to consider that the flow probes measure only local CBF and do not provide perfusion measurements of the entire cerebral hemisphere. Although the manufacturers state that accurate measurements of blood flow are possible within a $0.3 \mathrm{~cm}^{3}$ volume surrounding the probe, studies have shown that the flow probe yields an accurate reflection only of very localized blood flow. ${ }^{12,13}$ This is another potential limitation of using the perfusion values obtained with these probes to guide postoperative blood pressure management. Therefore, additional data from imaging studies may be used as adjuncts to inform such management.

\section{Conclusions}

Our observations show that episodes of local hypoperfusion clinically correlate with TNEs. Our results suggest 


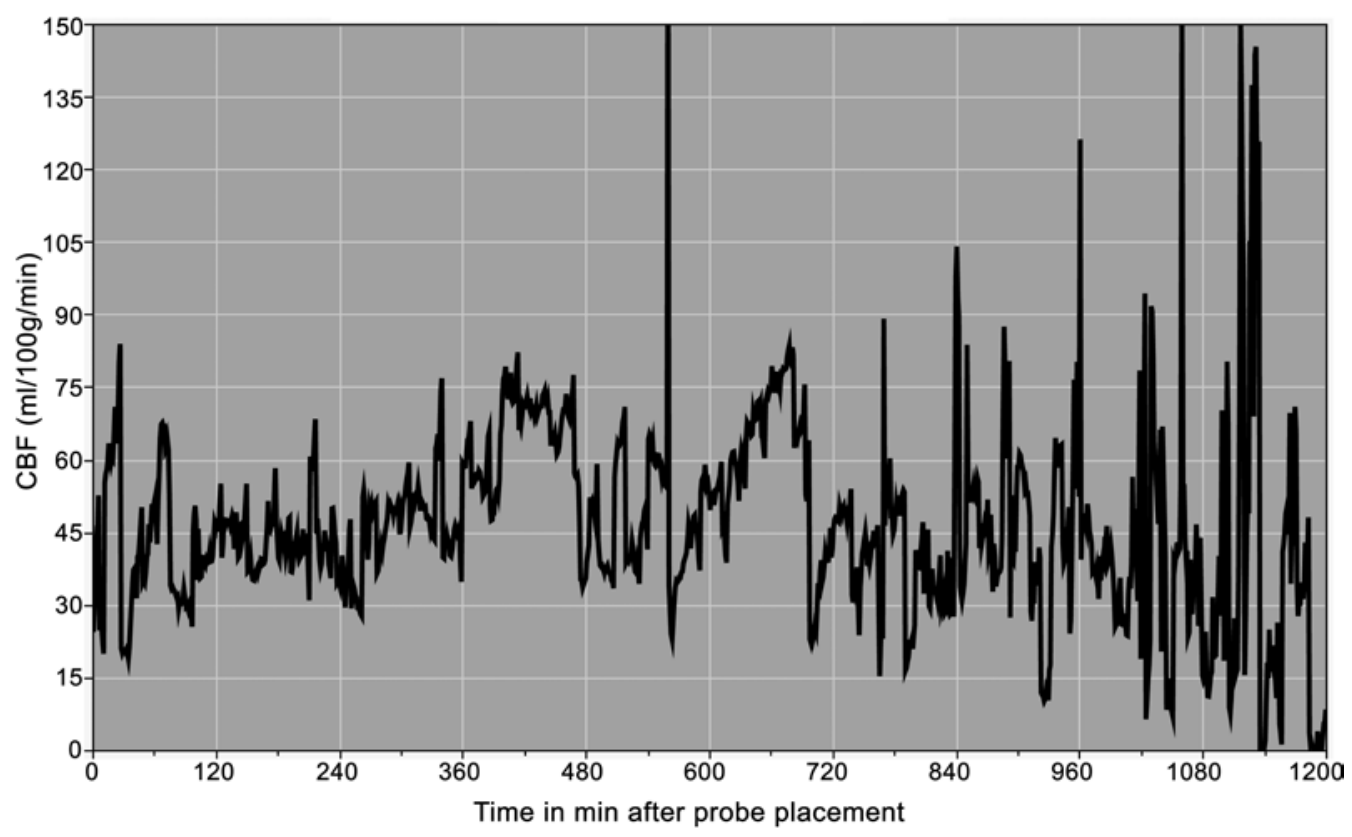

FIG. 2. A snapshot of the raw, unsmoothed time-series data, demonstrating the high degree of variability and need for smoothing methods. Data are from a Group 1 patient.

that managing blood pressure, closely guided by real-time perfusion data, may decrease the incidence of TNEs in these patients. Additional studies with larger numbers of patients are required to further validate this hypothesis.

\section{Acknowledgments}

We thank Cindy H. Samos for assistance with the manuscript and Beth Hoyte for assistance with the figures.

\section{References}

1. Fujimura M, Kaneta T, Mugikura S, Shimizu H, Tominaga T: Temporary neurologic deterioration due to cerebral hyperperfusion after superficial temporal artery-middle cerebral artery anastomosis in patients with adult-onset moyamoya disease. Surg Neurol 67:273-282, 2007

2. Fujimura M, Kaneta T, Shimizu H, Tominaga T: Symptomatic hyperperfusion after superficial temporal artery-middle cerebral artery anastomosis in a child with moyamoya disease. Childs Nerv Syst 23:1195-1198, 2007

3. Fung LWE, Thompson D, Ganesan V: Revascularisation surgery for paediatric moyamoya: a review of the literature. Childs Nerv Syst 21:358-364, 2005

4. Guzman R, Lee M, Achrol A, Bell-Stephens T, Kelly M, Do HM, et al: Clinical outcome after 450 revascularization procedures for moyamoya disease. Clinical article. J Neurosurg 111:927-935, 2009

5. Guzman R, Steinberg GK: Direct bypass techniques for the treatment of pediatric moyamoya disease. Neurosurg Clin $\mathbf{N}$ Am 21:565-573, 2010

6. Jaeger M, Soehle M, Schuhmann MU, Winkler D, Meixensberger J: Correlation of continuously monitored regional cerebral blood flow and brain tissue oxygen. Acta Neurochir (Wien) 147:51-56, 2005

7. Kobayashi H, Maeda H, Hayashi M, Kawano H, Handa Y, Kabuto M, et al: Assessment of cerebral perfusion in EC-IC bypass patients. Zentralbl Neurochir 52:41-43, 1991

8. Lee M, Guzman R, Bell-Stephens T, Steinberg GK: Intraop- erative blood flow analysis of direct revascularization procedures in patients with moyamoya disease. J Cereb Blood Flow Metab 31:262-274, 2011

9. Narisawa A, Fujimura M, Shimizu H, Tominaga T: [Seizure following superficial temporal-middle cerebral artery anastomosis in patients with moyamoya disease: possible contribution of postoperative cerebral hyperperfusion.] No Shinkei Geka 35:467-474, 2007 (Jpn)

10. Ohue S, Kumon Y, Kohno K, Watanabe H, Iwata S, Ohnishi T: Postoperative temporary neurological deficits in adults with moyamoya disease. Surg Neurol 69:281-287, 2008

11. Pandey P, Steinberg GK: Neurosurgical advances in the treatment of moyamoya disease. Stroke 42:3304-3310, 2011

12. Rosenthal G, Sanchez-Mejia RO, Phan N, Hemphill JC III, Martin C, Manley GT: Incorporating a parenchymal thermal diffusion cerebral blood flow probe in bedside assessment of cerebral autoregulation and vasoreactivity in patients with severe traumatic brain injury. Clinical article. J Neurosurg 114:62-70, 2011

13. Vajkoczy P, Horn P, Thome C, Munch E, Schmiedek P: Regional cerebral blood flow monitoring in the diagnosis of delayed ischemia following aneurysmal subarachnoid hemorrhage. J Neurosurg 98:1227-1234, 2003

14. Zipfel GJ, Fox DJ Jr, Rivet DJ: Moyamoya disease in adults: the role of cerebral revascularization. Skull Base 15:27-41, 2005

\section{Author Contributions}

Conception and design: Steinberg, Mukerji. Acquisition of data: all authors. Analysis and interpretation of data: all authors. Drafting the article: Mukerji. Critically revising the article: all authors. Reviewed submitted version of manuscript: all authors. Approved the final version of the manuscript on behalf of all authors: Steinberg. Statistical analysis: Mukerji.

\section{Correspondence}

Gary K. Steinberg, Department of Neurosurgery, Stanford University School of Medicine, 300 Pasteur Dr., R-281, Stanford, CA 94305-5327. email: gsteinberg@stanford.edu. 